\title{
Unsuccessful remembering: A challenge for the relational view of memory
}

\author{
André Sant'Anna* \\ Forthcoming in Erkenntnis
}

\begin{abstract}
This paper explores the relationship between a prominent version of the relational view of memory and recent work on forms of unsuccessful remembering or memory errors. I argue that unsuccessful remembering poses an important challenge for the relational view. Unsuccessful remembering can be divided into two kinds: misremembering and confabulating. I discuss each of these cases in light of a recent relational account, according to which remembering is characterized by an experiential relation to past events, and I argue that experiential relations do not adequately distinguish between successful and unsuccessful remembering. This is because there are, on the one hand, cases of remembering that do not instantiate the relevant experiential relations, and, on the other hand, cases of confabulation and misremembering that do instantiate the relevant experiential relations. I conclude by suggesting that any successful relationalist attempt to explain remembering needs to come to grips with unsuccessful remembering.
\end{abstract}

Keywords: Relationalism; Misremembering; Confabulating; Memory errors; Episodic memory.

${ }^{*}$ Centre for Philosophy of Memory / Institut de Philosophie de Grenoble, Université Grenoble Alpes. Email: rosolemandre@gmail.com 


\section{Introduction}

While a historically prominent view, relationalism about memory has only recently begun to be explored by contemporary philosophers of memory. In a relatively recent paper, Debus (2008) has attempted to revive interest in relationalism about memory by developing a version of the view in a more contemporary setting. According to the relational view of memory offered by her, recollective remembering, or memory of particular events or objects, is fundamentally a matter of being experientially related to those events or objects. This experiential relation is said to put us in a more basic relation of acquaintance with the things remembered, such that they become constitutive parts of our memories. To illustrate with an example, when I remember my college graduation, the relationalist says, I am acquainted with the event of my college graduation, such that it would impossible for me to have a memory of this event, or to individuate it as such, had the event not occurred $(2008,415)$.

The relational view can be motivated on at least two different grounds. The first is epistemological: the relational view, Debus says, "must be true if we accept (as we should) that people can sometimes gain knowledge about the past on the basis of their [recollective memories]" (Debus 2008, 406-7). The second motivation is semantic: the relational view "must be true if we accept (as we should) that subjects can form certain beliefs about the past on the basis of an [recollective memories], and that they can understand the propositions they endorse in holding on to the relevant beliefs" (Debus 2008, 407). The basic thought here is that, to account for the fact that we sometimes gain knowledge of the past, and for the fact that we form beliefs about the past - and understand those beliefs - we need an account of recollective memory in which it puts us in a relation of acquaintance with the relevant past events or objects.

Despite these motivations, the relational view has been met with skepticism by philosophers of memory. One common criticism that is often raised as soon as one begins to speak of a relational approach to memory is that it is simply wrong to characterize recollective memory in terms of an experiential relation to the past. While, following relationalists or naïve realists about perception (Campbell 2002; Martin 2004; Fish 2009; Brewer 2011), it may seem natural to think of perception as an experiential relation to events and objects, and while it may be tempting to draw an analogy between perception and memory-indeed, Debus is very clear on the fact that her relational account is inspired by relational or naïve realist accounts of perception-it is a mistake, the objection goes, to think of memory as an experiential relation to, or a form of acquaintance with, past objects or events. This is because, unlike in perception, where the objects perceived almost always co-exist with perceptual experiences, and as such, they are the kind of thing that one can be acquainted with, the objects of memory have ceased to exist at the time in which one remembers, and hence are not the kind of thing that one can be acquainted 
with. Either the relational view commits to some form of eternalism (e.g., Bernecker 2008), or the kind of relation involved in recollective memory is not an experiential relation.

For my purposes in this paper, I will set this line of criticism aside, as it has been pursued elsewhere in the literature (Michaelian 2016a; Sant'Anna 2018a; Sant'Anna and Michaelian 2019, and will grant the relationalist that there is a plausible way to conceive of memory as being an experiential relation to past events. I will focus here on a different line of criticism to the relational view that is based on the possibility of unsucessful remembering, or memory errors. By extracting an analysis of remembering from the relational view developed by Debus (2008), I will argue that it cannot properly distinguish between successful occurrences of rememberingwhen we remember an event that happened - from unsuccessful occurrences of rememberingwhen we seem to remember an event that happened, but incorrectly (misremembering), or when we seem to remember an event that did not happen (confabulating). The focus on unsuccessful remembering, or memory errors, will allow us to evaluate the prospects of the relational view in relation to recent developments in philosophy of memory.

I proceed as follows: In Section 2, I start by discussing the relational view of memory as developed by Debus (2008). In Section 3, I move on to outline an analysis of remembering extracted from the relational view. In Section 4, I introduce the idea of unsuccessful remembering and argue that the relational view, as formulated by Debus (2008), is unable to distinguish appro-

priately between successful and unsuccessful remembering. Section 5 concludes by addressing a few objections to the main argument.

\section{The relational view of memory}

The relational view developed by Debus (2008) says that what is characteristic of remembering is that subjects stand in an experiential relation to the past events. The term "experiential relation" is a technical one, and since it is central to her account, it will be helpful to examine it more carefully. As Debus points out at the beginning of her paper, she is concerned with recollective memories, which, according to her, are memories that have experiential characteristics. Recollective memories are, roughly speaking, equivalent to what psychologists call today episodic memories (see Tulving 1972, 1985). In other words, they are memories of particular events that one experienced in the past, such that, when one remembers, it seems to one that one is "re-experiencing" or "re-living" the relevant event (Tulving 2002). Thus, those memories have similar characteristics to sensory perception, in the sense that we seem to "see", "hear", "smell", and so on, the relevant features associated with the original event, but now "inside" our minds.

Being able to "re-experience" or "re-live" an event requires, in turn, that one experienced 
the relevant event in the past. It is important to note that, when one remembers, one is not literally having another perceptual experience of the event, but is rather undergoing a different kind of mental state that has a distinctive relationship to the past event, namely, a specific kind of experiential relationship. So, while it is true that both perception and memory have experiential characteristics, and while it is arguably the case that both put us in an experiential relation to their objects, the relevant experiential relation instantiated in each case is different. For Debus, the experiential relation instantiated in the case of memory is characterized as one that supervenes on specific causal, temporal, and spatial relations that hold between subjects and the past events. The relation is causal because, as she points out, when one remembers, certain neurophysiological events occur in one's brain. Moreover, these events are caused by earlier neurophysiological process that were, in their turn, ultimately caused by the past events at the time of the original experience (Debus 2008, 411). Similarly, the relation supervenes on spatial and temporal relations because, as I mentioned initially, remembering requires that the subject experienced the relevant event before. That is, the original experience must happen earlier than the memory and there must be a causal connection with the original experience. ${ }^{1}$

In addition to the claim that memory requires a specific experiential relation to the past, which Debus calls the Relation Claim, the relational view of memory requires us to make two additional claims about the nature of recollective memories: the Constitution Claim $(2008,415)$ and the Consciousness Claim (2008, 421). The Constitution Claim is the claim that past events themselves are constitutive parts of mental occurrences of remembering. Thus, for a subject to remember his tenth birthday party, it is necessary to make reference to his tenth birthday party in order to individuate this memory as a memory of that particular event. Having a memory as of his tenth birthday party — which may or may not have occurred-does not, according to this view, suffice for the subject to count as genuinely remembering his tenth birthday party in the same sense that, for relational views of perception, having a perceptual experience as of a bird sitting on a fence does not suffice for a subject to count as perceiving the birdwhich may or may not be out there. The Consciousness Claim is, in contrast, the claim that past events themselves are "constituent[s] of the conscious recollective experience itself" (2008, 406, author's italics). According to Debus, "[t]his means that, when a subject [recollectively remembers] a past object (or event), the past object (or event) is, for the [remembering] subject, immediately available in consciousness." $(2008,406)$. It is not entirely obvious what she has in mind here, but the thought seems to be that recollective memories are transparent (Harman

\footnotetext{
${ }^{1}$ Debus frames this idea in terms of the original experience and the memory being in the same "spatiotemporal path" traced by the subject in the world. According to her, by inhabiting the world, subjects trace a certain "spatiotemporal path", and to count as remembering something, the spatiotemporal point in which the memory occurs must precede the spatiotemporal point in which the experience occurred, and both must belong to the same "spatiotemporal path".
} 
1990; Tye 2000), namely, when one remembers, one is not consciously aware of the properties of one's conscious experience, but rather of the properties of the objects of awareness themselvesin the case of recollective memory, the properties of the past events or objects themselves.

These three claims - the Relation Claim, the Constitution Claim, and the Consciousness Claim - sit at the core of the relational view of memory, and they help us to see more clearly what the relational view is a view of. In addition to being a claim about the objects of memoryi.e., that memory is of past events, and hence that one needs to be related to those events (causally or otherwise) in order to count as remembering - it is also a claim about the nature of memory as a mental state. In other words, it is claim about the metaphysics of memory-i.e., what it takes to individuate a mental state as a recollective memory of an event - and a claim about the phenomenology of memory-i.e., what it is like to recollectively remember an event. While Debus does not say anything explicit to this effect, her heavy reliance on naïve realist and disjunctivist approaches to perception $(2008,408 ; 414)$, such as the ones developed by Martin (2004) and Campbell (2002), suggests that her relational view tries to avoid committing to the presence of representations to account for memory as a mental state. ${ }^{2}$ While, in general, representationalists would agree that the objects of recollective memory are indeed past events, they would deny that such a relation is established by an experiential relation along Debus's line (see Bernecker 2008; Robins 2016b; Michaelian 2016c; Sant'Anna 2018a; Fernández 201x). Instead, what characterizes a mental state as a state of recollective memory is, for the representationalist, the fact that it puts us in an intentional relation to the past, that is, a relation of representing those events. ${ }^{3}$ Thus, to illustrate with an example, what makes my memory of my tenth birthday party a memory of that event, and what accounts for the fact that I experience that memory as a memory of

\footnotetext{
${ }^{2}$ For a more detailed discussion of why relational views of perception are incompatible with representational views, see Crane (2006); Genone (2016); Locatelli and Wilson (2017). In what follows, I will take it for granted that one of the motivations for developing a relational account of memory is to avoid a representationalist account. As mentioned above, it is true that Debus (2008) does not discuss this opposition in her paper, but I take it that, in addition to her heavy reliance on the naïve realist/relationalist literature in perception, the absence of any reference to 'representations', 'content', 'intentional properties', 'accuracy conditions', and other similar terms that have been systematically utilized by representationalists allows us to safely conclude that she intends her relational view as an alternative account to representationalist accounts, even if she does so only implicitly. One may, of course, plausibly argue that her view is committed to the presence of representations, but since Debus (2008) is silent on this issue, settling it would require a much closer exegetical analysis of her work, a task that is beyond the scope of this paper.

${ }^{3}$ It is important to note that representationalism, at least as understood in the context of the literature discussed here, is only committed to the claim that memory is a representational state at the personal level. This is what it means to say that representationalism and relationalism are opposing accounts of memory as a mental state. More recently, some have argued that memory is not representational in the sense that the subpersonal processes responsible for producing memories (understood as mental states) do not involve the manipulation of representations (e.g., Hutto and Myin 2017; Hutto and Peeters 2018; see Michaelian and Sant'Anna 2019 for discussion). The issue of whether memory understood as a mental state is representational is, as these authors recognize, independent of whether memory processes involve the manipulation of representations. Thus, whether or not a view is committed to the idea that memory processes involve the manipulation of representations at the subpersonal level is tangential to the issue separating representationalism and relationalism in the way that these views are understood here.
} 
that event, is that it represents my tenth birthday party, and not that it is experientially related to, and hence is constituted by, that event. Given Debus's three claims and her reliance on the relationalist literature in perception, the relational view of memory can plausibly be seen as a competing account to representationalism of the nature of memory as a mental state. ${ }^{4}$

In what follows, I will not be concerned with the Constitution Claim and the Consciousness Claim, but only with the Relation Claim. For this reason, the argument that I will develop against the relational view will remain neutral on whether the relational view provides a compelling account of recollective memory as a mental state. My aim in this paper is more modest: I will discuss in more detail the question of when, for the relational view, a subject can be said to be genuinely remembering a past event. In other words, I will focus, from now on, on the question of what an analysis of remembering is according to the relational view.

\section{The relational analysis of remembering}

While there are some indications of what a relational analysis of remembering is supposed to be following the relational view, Debus does not develop this point in much detail. ${ }^{5}$ For this reason,

\footnotetext{
${ }^{4}$ While some may view the argument developed in the paper as an implicit endorsement of representationalism, I shall not take a firm stand on the subject here. Recent work on the dispute between representationalism and relationalism in philosophy of perception suggests that they are not mutually exclusive views and hence that "hybrid" views are possible (see Schellenberg 2010, 2011; Siegel 2010; Logue 2014; Hanna 2015; Sant'Anna 2018b). In fact, in a recent paper, Sant'Anna (2018a) has made a first attempt to propose a hybrid account of memory along these lines. Another promising approach would be to think about hybrid views in relation to the distinction between conceptual and nonconceptual content (see Russell and Hanna 2012; Hanna 2015). In particular, the notion of nonconceptual content developed by Hanna (2015), which sits at the core of his hybrid account of perception, seems to provide a promising starting point for hybrid approaches to memory. This is because, once we understand spatiotemporal representations in episodic memory within a Kantian framework, as has been suggested by Russell and Hanna (2012) and Hanna (2015), we can have a view that avoids various pressing problems for relational approaches to memory, such as the co-temporality problem and the problem of how we can be directly aware of the past (see Bernecker 2008; Sant'Anna and Michaelian 2019), while at the same time preserving core relationalist and representationalist claims. Since, however, hybrid approaches still continue to be largely unexplored in the memory literature, I will not discuss them any further.

${ }^{5}$ In a more recent paper, Debus (2010) offers an analysis of remembering that tries to supplement the analysis offered by the causal theory of memory (see footnote 6) by adding an extra criterion, not present in the causal theory itself, according to which a subject counts as remembering only if the memory representation is epistemically relevant for the subject-i.e., the subject believes that he is remembering. In the following discussion, I will not appeal to the Debus's 2010 paper in my attempt to outline an analysis of remembering in line with the relational view for two reasons. The first is that it is not obvious how the 2010 paper relates to the 2008 paper, which is the focus of my discussion. As I pointed out before, there are good reasons to view the 2008 paper as an attempt to provide a non-representational account of memory. However, due to its close reliance on the analysis offered by the causal theory of memory, which has often been interpreted as a representational account—and, in my view, rightly so, for one of the conditions it ascribes to remembering makes explicit reference to accuracy conditionsthe 2010 paper seems to be in tension with the overall proposal of the 2008 paper. It may be that they belong to two different projects, or that Debus simply changed her mind, but since it is beyond my scope to provide a comprehensive criticism of her work, I will not speculate any further here. The second reason why the 2010 paper will not be further discussed is because, even if we were to set aside the worries about the coherence of the views
} 
I propose that we start by looking at the analysis offered by the causal theory of memory, ${ }^{6}$ which is the dominant theory in the literature, and then modify it appropriately to fit with the relational view. Before proceeding, it is important to note that the causal theory and the relational view are engaged in two distinctive projects, which are themselves constrained by different considerations. The causal theory is ultimately an attempt to offer an analysis of remembering. The relational view is, in contrast, an attempt to offer an account of the nature of memory as a mental state. Thus, in what follows, I will discuss an analysis of remembering that takes the relational account of memory as a mental state as a starting point.

According to Martin and Deutscher (1966), who provided the original formulation of the causal theory, a subject $S$ will count as remembering an event $e$ iff:

(1) $S$ has previously experienced $e$;

(2) $S$ has a present mental representation of $e$;

(3) $S$ stands in an appropriate causal relationship to $e$;

(4) The content of $S$ 's present representation is sufficiently similar to the content of $S$ 's previous experience. ${ }^{7}$

To illustrate, consider my putative memory $\mathrm{M}$ of my tenth birthday party. According to Martin and Deutscher, $\mathrm{M}$ is an instance of remembering because: (1) I had a previous experience of my tenth birthday party, which led me to form representations of this event; (2) I have a current representation $\mathrm{M}$ of my tenth birthday party; (3) my actual representation $\mathrm{M}$ is caused, perhaps by means of a memory trace, by my past experience of the party; and (4) the content of $\mathrm{M}$ is sufficiently similar to the content of the past experience-e.g., I remember my parents being there, I remember having chocolate cake, and so on, which were all represented in my previous perceptual experience of the party. (1)-(4) are, therefore, necessary and jointly sufficient conditions to say that a subject count as remembering.

Now, can (1)-(4) be incorporated into a relational analysis of remembering? Let's consider (1) first. Since Debus says quite explicitly that successful remembering requires that subjects have experienced the relevant events in the past, and this is central to make sense of the notion of an experiential relation, it seems that (1) can be straightforwardly incorporated into the relational

defended in the 2008 and the 2010 paper, the novel condition discussed by the 2010 paper-the epistemic relevance condition - would not help addressing the problems raised later on in the paper, for all the cases discussed there are epistemically relevant for the subject.

${ }^{6}$ For the original formulation of the causal theory, see Martin \& Deutscher (1966). For more recent developments, see Bernecker (2010) and Cheng and Werning (2016). For helpful discussions, see Robins (2016b) and Michaelian and Robins (2018)

${ }^{7}$ This formulation is adapted from Bernecker (2010, ch. 1). See also Bernecker $(2015,302)$. 
analysis. The same is true of (3). That is, the experiential relation is said to supervene on causal and spatiotemporal relations holding between subjects and events. However, (2) and (4) do not seem compatible with the relational view. As I pointed out before, Debus (2008) is not very explicit on whether or not she thinks that remembering can involve some sort of representation. However, there are good reasons to think that it does not. The first reason is that, later on in the paper, Debus says that experiential relations are supposed to make subjects directly aware of the past events - this is the Consciousness Claim discussed above. So, when one remembers, it is not the case that one's awareness of the event is mediated, which eliminates the need for representations. ${ }^{8}$ The second reason is that Debus frames her view in relation to Martin's (2004) relational view of perception, which avoids representations altogether. While Debus says quite explicitly that her understanding of mnemonic awareness differs in important respects from what is usually understood by perceptual awareness - although she says very little about what the differences are supposed to be-it looks like that the same, or at least very similar, motivations drive both the relational view of memory and relational views of perception. This does not, of course, make a conclusive case against the presence of representations, but it gives us good reasons to cast doubt on the inclusion of (2) and (4) into the relational analysis.

Thus, by looking at the analysis proposed by Martin and Deutscher (1966), we can use (1) and (3) as starting points to conceive of a relational analysis of remembering. Considered alone, however, (1) and (3) provide only a partial picture of a relational analysis. The reason is that, for the relational view, the relationship that subjects have to past events is not merely causal. In order to provide a more complete picture, the relational analysis needs to require that subjects stand in an experiential relation to the relevant events, which takes into account not only a causal relation, but spatial and temporal relations as well. On this view, then, the relational view of memory offered by Debus seems to suggest that $S$ remembers $e$ iff:

(R1) $S$ has previously experienced $e$;

(R2) $S$ is presently experientially related to $e$ by undergoing a mental state $M$; where the experiential relation supervenes on specific causal, spatial, and temporal relations obtaining between $S$ and $e$.

To illustrate with the same example as above, the relational view says that I remember my tenth birthday party because (R1) I had a previous perceptual experience of the party, and (R2) I am experientially related to the party in the sense that my memory is caused by it and the party is located at a spatiotemporal point that precedes the actual spatiotemporal point in which I have

\footnotetext{
${ }^{8}$ Although see Bernecker (2008, ch. 5), who claims that the presence of representations in memory is not incompatible with a form of direct realism. I will leave this issue aside as it is not clear whether Bernecker's view is a genuine form of direct realism.
} 
the memory. On this view, then, (R1) and (R2) are viewed as necessary and jointly sufficient conditions to say that a subject count as remembering.

One important clarification at this point refers to the nature of the mental state $M$ in (R2). Since, on the relational view, $\mathrm{M}$ cannot be understood as a representational state, one natural question would be what $\mathrm{M}$ is according to the relational view. The traditional move by relationalists in perception is to understand $\mathrm{P}$ - the non-representational state counterpart of $\mathrm{M}$ in perception - as being a more basic form of acquaintance with particular objects in the external world, such that those objects become constitutive parts of perceptual experiences (see Martin 2004; Fish 2009; Brewer 2011). As Fish puts it nicely, the objects "shape the contours of the subject's conscious experience by actually being the contours of the subject's conscious experience" $(2009,6)$. Similarly, in the case of memory, one might understand M as a more basic form of acquaintance with the past, in which the past events become constitutive parts of mnemonic states. This seems to be what Debus has in mind when she talks about memory making us directly aware of the past, although, I should note again, she is careful to make explicit that the kind of awareness involved in memory differs in important senses from the kind of awareness involved in perception (see Debus 2008, 408). ${ }^{9}$

Now, the relational analysis stated above is intended as a response to the general question of what is it to remember, as opposed to, e.g., the questions of what is it to perceive or what is it to imagine. However, a complete theory of remembering should not only distinguish between remembering and other mental phenomena, but also adequately distinguish between different ways in which remembering can occur. And here the distinction between successful and unsuccessful occurrences of remembering becomes crucial. ${ }^{10}$ Successful remembering refers to, roughly speaking, cases where we remember things as they happened. Unsuccessful remembering, in contrast, can take two different forms. The first is misremembering (Robins 2016a),

\footnotetext{
${ }^{9}$ One worry that one may have here is that relying on Martin and Deutscher's (1966) classical analysis is unfair to the relationalist. This is because Martin and Deutscher's view is allegedly a representational one and, as such, the analysis provided by them is bound to be incompatible with relationalism. In response, I would like to make it clear that the reasons for relying on Martin and Deutscher's analysis are two: first, it is by far the most prominent analysis of remembering in the recent literature, so it has shaped virtually all subsequent attempts at such an analysis; and second, and more importantly, alternative causal theories of remembering, including Debus's (2008) relational account, have relied on Martin and Deutscher's account more or less systematically (see Michaelian and Robins 2018 for discussion). More to the point, though, the fact that Martin and Deutscher's original analysis is a representational analysis does not harm my current project. Condition (R1), which is the same as Martin and Deutscher's condition (1), and condition (R2), which is the relational version of Martin and Deutscher's condition (3), are nonrepresentational conditions, and consequently, they do not require any commitment to representationalism-the representational conditions, (2) and (4), have been consistently rejected precisely because they rely on a form of representationalism. Moreover, the motivation for endorsing conditions (R1) and (R2) do not come from some veiled commitment to Martin and Deutscher's original theory; instead, these conditions reflect central claims of the relational view espoused by Debus, as was discussed in more detail above.

${ }^{10}$ At this point, some relationalists might want to object that they do not need to be concerned with this, as what I am calling "unsuccessful remembering" is not actually a form of remembering, but a mental state of another kind entirely. I address this objection in more detail in the next section.
} 
which corresponds to cases where we get some details of an event right, but get some other details of an event wrong. ${ }^{11}$ The second is confabulation (Hirstein 2005; Michaelian 2018; Robins 2018), which corresponds to cases where we get all the details wrong-i.e., the event remembered did not happen. Since the presence of an experiential relation is central to the relational analysis, one would expect that its presence or its absence would provide a meaningful way to distinguish between successful and unsuccessful remembering. In fact, this seems to be the suggestion behind Debus's (2008) overall proposal. However, it is not entirely clear whether this will work. In the next section, I will argue that (R2), or the requirement for the presence of an experiential relation, is neither necessary nor sufficient to distinguish successful and unsuccessful remembering, and thus that the relational analysis, as formulated in here, is at best incomplete.

\section{Unsuccessful remembering}

The problem of unsuccessful remembering and its relationship to the relational view of memory view has not been explored in much detail. In the perception literature, however, forms of what we might call unsuccessful perception-i.e., illusions and hallucinations-have proven to pose real challenges for relational views of perception (see Smith 2002; Crane and French 2017). The main reason for this is that successful or veridical occurrences of perception can be indistinguishable, from the point of view of the subject, from unsuccessful or non-veridical ones. Thus, when relationalists say that perception is an experiential relation to the perceived objects, they need to explain how and why there can be phenomenologically indistinguishable perceptual experiences both in the presence and in the absence of the objects perceived.

The same problem, I think, arises for relational views of memory. Successful and unsuccessful remembering can often be phenomenologically indistinguishable, which questions the necessity of there being an experiential relation to past events when one remembers. In the same way in which I can seemingly see a spider on the wall and fail to realize that there is not a spider on the wall, I can seemingly remember an event, e.g., my tenth birthday party, and fail to realize that this event did not happen. However, if I can seemingly remember an event when it did not occur, and hence undergo the same mental state as the one I would have undergone had the event

\footnotetext{
${ }^{11}$ I should say that this is not necessarily what Robins understands by misremembering. For Robins, "[m]isremembering is a memory error that relies on successful retention of the targeted event" (2016a, 433), which is paradigmatically illustrated by the DRM effect, where the content of what is misremembered is non-trivially related to the content of what is remembered or retrieved-e.g., subjects are more likely to misremember seeing particular fruits, such as bananas, if the content of what is remembered or retrieved also involves fruits, such as apples and oranges. The definition of misremembering that I am using here does not require such a non-trivial relation. According to this definition, one misremembers if the content of what is misremembered was not originally experienced.
} 
been the case, then it does not seem to make sense to postulate a relationship to such event as necessary for remembering. That is, the fact that one can seemingly remember an event when the event did not happen "screens off" (Martin 2004) any explanatory role played by the past events in one's theory of memory.

One strategy popular among relationalists about perception is to resort to a disjunctivist view of perception. There are different varieties of disjunctivism (see Byrne and Logue 2008), but one popular characterization is that, at the most fundamental level, successful or veridical and unsuccessful or non-veridical perceptual experiences do not share any essential features. In other words, they are viewed as occurrences of two different kinds (see Snowdon 1980; Martin 2004; Fish 2009). Thus, if disjunctivism is true, it is no longer a problem to say that veridical perceptual experiences are relational, while non-veridical ones are not. In her discussion of "memory hallucinations", which I have called "confabulations" here, Debus (2008) employs the same strategy. Inspired by Martin's (2004) disjunctivist account of perception, she says that the fact that two mental occurrences are indiscriminable from a subjective point of view does not imply that they are mental occurrences of the same kind. For Debus, the absence of the experiential relation in the case of the so-called memory hallucinations postulates a fundamental separation between them and successful remembering $(2008,414)$. This explains why, despite being phenomenologically indistinguishable, successful memories are relational and memory hallucinations, or confabulations, are not.

Thus, if combined with disjunctivism, the relational analysis can provide a principled way to distinguish successful remembering from at least one kind of unsuccessful remembering, namely, what Debus calls "memory hallucinations", or what I have called "confabulations". However, I think that, even with disjunctivism, the relational analysis fails to provide a satisfactory way to distinguish between successful and unsuccessful remembering. The main reason, as I will argue in section 4.1 , is that memory hallucinations or confabulations do satisfy (R2) above, so there is no principled way to say that they are fundamentally distinct from successful remembering. But before I turn to this question, let me define more precisely what I will understand by unsuccessful remembering. As I said before, unsuccessful remembering can take two forms. The first form, misremembering, is when we remember incorrectly one or more details of an event that occurred. The second form, confabulation, is when we remember an event that did not occur at all.

To illustrate, consider misremembering first. Consider, for instance, my memory of my tenth birthday party. I remember my parents being there, I remember it being a sunny day, and I remember having chocolate cake. While this memory is about an event that happened, at least one of its elements incorrectly describe the event; that is, it was a rainy rather than a sunny day. So, despite being about an event that happened, and despite getting some details right-i.e., my 
parents were there and I had chocolate cake-I mis-remember the event in question. Consider confabulations now. Suppose that I remember being to Moscow when I was a teenager. Since I have never been to Russia, this memory will count as an occurrence of confabulation because the event in question did not happen. ${ }^{12}$

Now that we have a clearer understanding of what unsuccessful remembering is, I will move on to show why it poses a problem for the relational view of memory. Section 4.1 will discuss confabulations and Section 4.2 will discuss misremembering. My main argument will be that both forms of unsuccessful remembering instantiate the relevant experiential relation to past events and hence that the relational analysis of remembering fails to properly distinguish them from successful remembering.

\subsection{The problem of confabulation}

Can the relational analysis of remembering rule out cases of confabulations? To answer this question, we should go back to (R1) and (R2) above. For the relational view, $S$ remembers $e$ iff:

(R1) $S$ has previously experienced $e$;

(R2) $S$ is presently experientially related to $e$ by undergoing a mental state $M$; where the experiential relation supervenes on specific causal, spatial, and temporal relations obtaining between $S$ and $e$.

The question is, then, whether there are cases of confabulation that satisfy (R1) and (R2). The most obvious way to rule out cases of confabulation would be to appeal to (R1). Since the events that one confabulates have not happened, it is not possible that one experienced those events in the past. Therefore, confabulations do not satisfy (R1) and are thus distinct from occurrences of successful remembering. While I think this provides a satisfactory way to deal with some cases of confabulations, ${ }^{13}$ it does not seem to reflect the motivation behind the relational view. When she introduces disjunctivism to deal with confabulations, Debus argues that confabulations are distinct in kind from successful remembering because, in the former case, the relevant experiential relationship fails to obtain. So, at least on Debus's version of the relational view, it is the

\footnotetext{
${ }^{12}$ With the exception of a few remarks about "memory hallucinations", Debus (2008) says very little about memory errors, so the criticism that I will present in the following sections relies mostly on a characterization of unsuccessful remembering at play in recent debates in the philosophical literature. How to characterize memory errors and how to account for them are lively and controversial questions in the current literature, so I shall not take a stand on them, but the basic distinction between misremembering and confabulating utilized here seems to be relatively uncontroversial. For more discussions about misremembering and its relationship to current theories of remembering, see Robins (2016a) and Michaelian (2016b). For discussions about confabulation in particular, see Hirstein (2005), Robins (2018), and Michaelian (2018), and for discussions about the relationship of confabulation to current theories of remembering, see Michaelian (2016b; 2018), Bernecker (2017), and Robins (2017; 2018).

${ }^{13}$ As I will argue below, it fails to account for cases of veridical confabulations (Michaelian 2016b).
} 
presence of (R2), and not of (R1), that provides the means to distinguish confabulations from successful remembering.

I will come back to (R1) later. For now, let us focus on (R2), as it is the central element of Debus's view. The question now is, therefore, whether (R2) can provide a meaningful way to distinguish successful occurrences of remembering from confabulations. To start with, it is important to note that (R2) will be successful in providing such distinction only if we make the further assumption that, to successfully remember, subjects must be in a unique experiential relation to a unique past event. That being the case, the claim that confabulations do not count as successful remembering becomes straightforwardly true, for there is no unique experiential relation holding between the subject and the confabulated event, as the event in question did not happen. However, the relational view does not require us to make that assumption. The claim is simply that successful remembering instantiates the relevant experiential relation, while confabulations do not. Thus, if one does not make this assumption, it is not hard to envisage cases where confabulations instantiate experiential relations to past events in the way described by (R2).

To make this clear, let me introduce the notion of a partial experiential relation. ${ }^{14}$ A partial experiential relation is similar to the one described in (R2), that is, it supervenes on the causal, spatial, and temporal relations between a subject $S$ and a past event $e$. However, because of its partiality, the partial experiential relation only makes the subject aware of some but not all the constituents, or properties, of $e$. For example, I can be in a partial experiential relation to my tenth birthday party, such that I can remember having chocolate cake next to my parents, but fail to remember other elements, including that this memory is of my tenth birthday party. Suppose, now, that I remember an event that did not happen. Because the event did not happen, the relational view says that there cannot possibly be an experiential relation between the subject and the event, thus making this an occurrence of confabulation. But, it does not follow from this that the confabulation fails to put the subject in partial experiential relations to past events. It could be the case - and I will argue later that there are good reasons to believe that this is actually the case-that the confabulation puts the subject in two or more partial experiential relations to two or more past events, such that he becomes aware of different constituents or features of multiple events as belonging to a unique event. Consider, for instance, my memory of having pizza at the beach. While I have never experienced such an event, it is true that I have previously experienced the following two different events: one in which I had pizza and another one in

\footnotetext{
${ }^{14}$ I am not suggesting here that Debus (2008) herself is committed to partial experiential relations. While I believe that her view does not require us to commit to full experiential relations, I think that such a commitment is an underlying assumption of her proposal. However, as I will argue in what follows, the commitment to full experiential relations leaves relationalists with an overly restrictive account of remembering in light of research on the constructive character of remembering. Appealing to partial experiential relations, I will suggest, is the best way to avoid this worry in a relationalist framework.
} 
which I was at the beach. So, if the notion of a partial experiential relation is sound, there is no reason why I could not be aware of the confabulated event by being partially experientially related to two or more events that I experienced previously. ${ }^{15}$ However, if that is the case, then nothing prevents confabulations from putting us in experiential relations to past events.

One way for relationalists to avoid this problem would be to simply bite the bullet and add the additional clause that a mental state counts as successful remembering only if it makes the subject experientially related to a unique event. However, I think that this move should be resisted. If correct, it would lead to a restrictive, and hence counterintuitive, account of remembering. To see this, suppose that I have a putative memory $\mathrm{M}$ of my tenth birthday party, where I remember having chocolate cake with my parents and my friends. Since this event happened, and since I experienced it, (R1) is satisfied. However, M is not brought about by my being experientially related to the event of my tenth birthday party, but instead, by my being partially experientially related to different events (see Michaelian 2011; Robins 2016b). In this particular case, $\mathrm{M}$ puts me in a partial experiential relation to an event $e_{1}$, where I had chocolate cake, to an event $e_{2}$, where I had a meal with my parents, and to an event $e_{3}$, where I was hanging out with my friends. In other words, my awareness of the three distinctive constituents of M, namely, <having chocolate cake $>$, < having a meal with my parents $>$, < being with my friends $>$, are awareness of constituents of different past events that are brought together by $\mathrm{M}$.

If this is right, the claim that remembering requires a unique experiential relation will require us to say that I am not remembering my tenth birthday party. But this is problematic for at least two reasons. The first is that $\mathrm{M}$ above satisfies (R1) and, moreover, it is experientially related, in a relevant sense, to past experiences. From a common sensical point of view, this seems to be enough to say that one is successfully remembering, as one gets the relevant details right and one does so in virtue of being (partially) experientially related to the past. The only thing that is missing, arguably, is that the event that $\mathrm{M}$ purportedly makes me aware of, namely, my tenth birthday party, is not operative-i.e., it is not the ultimate cause-in producing M (see Martin and Deutscher 1966, p. 166). Thus, it might be argued that, despite seeming so, M does not count as a successful occurrence of remembering because none of the experiential relations trace back to the original event, which is precisely what is required to say that $\mathrm{M}$ allows me to be aware of my tenth birthday party.

This answer will, however, provide only a temporary solution. The reason is that, while the

\footnotetext{
${ }^{15}$ One worry here is that it is difficult to see how a subject could enjoy a single and unified conscious experience of remembering an event if multiple experiential relations constitute a single memory. For my present purposes, I am taking this for granted since, as it will become clear later, I think that appealing to partial experiential relations is the best strategy available for relationalists to make sense of the constructive character of remembering. However, if this turns out to be correct, then the question of how multiple experiential relations allow for a unified conscious experience in remembering will need to be properly addressed by relationalists. This point is discussed in more detail below.
} 
relational view might be able to rule out cases where none of the partial experiential relations trace back to the past event itself, it will not be able to rule out cases where at least one of the partial experiential relations establish such relation. Consider the case above again. Suppose that my partial awareness of $<$ having chocolate cake $>$ is, in fact, awareness of myself having chocolate cake in my tenth birthday party. However, my awareness of $<$ having a meal with my parents $>$ and of $<$ being with my friends $>$ are partial awarenesses of constituents of different events, i.e., $e_{2}$ and $e_{3}$. In this case, then, there is at least one experiential relation that traces back to the relevant past event, and thus $M$ should count as a successful occurrence of remembering even if there is more than one experiential relation connecting $M$ to multiple past events. Requiring the presence of a unique experiential relation is, therefore, too restrictive, which provides a counterintuitive account of memory.

So far, I have attempted to show that, from an intuitive point of view, requiring a unique experiential relation is implausible. However, relationalists might resist this by saying that they do not share our intuitions. To put it sharply, the claim here would be that, for relationalism, if there is not a unique experiential relation holding between the subject and the event remembered, then, intuitively, the subject's putative memory will not be an occurrence of successful remembering. This leads us to the second problem with the idea that remembering requires a unique experiential relation. While I think that this response is, in principle, open to relationalists, I do not think it is a promising one. The reason is that it provides an analysis of remembering that starts by stipulating what the phenomenon must be, instead of describing or explaining what the phenomenon actually is. The problem with this approach is that our theoretical intuitions do not always correspond to how the phenomenon actually is, which runs the risk of making our initial stipulations empty or explanatorily uninteresting.

And this, I think, is the problem with this strategy. In other words, it has been shown by empirical research that memory is constructive, in the sense that our memories are constructed from different sources other than the original experience (Schacter et al. 2007, 2012; Addis 2018). Some authors, such as Michaelian (2011), have suggested that while some causal link must be preserved to the past experience that the memory is about, not all the elements or constituents that figure into that memory need to be derived from the original experience. Such elements can come from different sources, such as semantic information (Cheng and Werning 2016; Cheng et al. 2016) or background knowledge (Suddendorf and Corballis 1997) present in retrieval (see also De Brigard 2014 and Michaelian 2016c). Based on this and other empirical findings, De Brigard (2014) has suggested, for example, that it is not unsurprising that most of the occurrences of remembering that we call "successful" will have elements that are not drawn from the original experience. Setting the details of this debate aside, the point is that how memory works, or how remembering happens in the world, does not seem to support the 
prior relationalist theoretical intuition that a unique experiential relation to the past event must be characteristic of successful remembering. If that was the case, most of the occurrences of remembering that we call "successful" would be actually unsuccessful. So, again, appealing to the presence of a unique experiential relation provides a restrictive, and hence inadequate, account of how memory works. Relationalists are thus faced with a dilemma: either they commit to full experiential relations, in which case they will end up with an overly restrictive account of remembering, or they commit to the notion of partial experiential relations, in which case their account would be too permissive, for confabulations would count as instances of remembering.

Before proceeding, I should pause to address two objections. The first says that acknowledging that memory is constructive and that multiple causal connections may influence memory retrieval does not require us to give up on the idea that remembering involves a unique experiential relation to a past event. This is because, the objection goes, construction and influence from multiple causal factors are not incompatible with the idea that a single past event, $e$, plays a continued role in producing memories of $e .{ }^{16}$ While this kind of response is available for causal theorists when dealing with problems raised by the constructive character of memory, it is not available for the relational view. This is because it is a mistake to interpret experiential relations as being mere causal relations in the sense used by causal theorists. For the causal theory, a causal relation is often established by means of a memory trace, which stores information from experience that is later retrieved to produce memory representations. Experiential relations do not, however, reduce to memory traces, or to any internal brain state for that matter. As Debus (2008, 419-20) points outs in her discussion of the Relation Claim, the causal relation between a memory and an event is just one of the elements that characterize an experiential relation, the other two being spatial and temporal relations. More importantly to my purposes, unlike memory traces as typically understood by causal theorists, experiential relations are supposed put us in a relation of acquaintance with the past events- that is, a conscious relation to the things that we are experientially related to. So, while, for the causal theory, it is possible for a subject to form a memory of a single event $e$ when multiple memory traces are operative in producing that memory, this is not possible in the context of the relational view unless one appeals to the notion of a partial experiential relation introduced earlier. Without the latter notion, however, one would end up with a view in which most of our memories would make us consciously aware of multiple events due to the presence of multiple experiential relations, which is clearly implausible.

The second objection says that, while it may be true that the relational view provides a restrictive view of memory in relation to empirical research, it does not necessarily follow that it is an inadequate one. Here, the relationalist could argue that an analysis of remembering may,

\footnotetext{
${ }^{16} \mathrm{I} ' \mathrm{~m}$ indebted to an anonymous referee for raising this objection.
} 
but need not, be aligned with an account of how the cognitive mechanisms underlying memory work. In essence, this is an instance of the more general—and highly controversial—question of how important empirical considerations are for philosophical analysis, a question that I do not expect to answer here. So, relationalists might well want to avail themselves of this strategy and claim, in response to the argument above, that it is not the business of philosophical analysis to try to make sense of empirical results, thus dismissing the argument that the relational view is restrictive and consequently inadequate. I think, however, that this is not a particularly promising line of response, for it is based on a highly controversial claim that will require substantial argumentation to be established. In saying this, though, I do not mean to endorse the equally controversial claim that philosophical analysis should be strictly about making sense of empirical results - a claim that also needs substantial argumentation to be established-but only the relatively uncontroversial and weaker claim that philosophical analysis must be broadly sensible to empirical results. Thus, once this latter claim is in place, the fact that a view is restrictive on the face of empirical results, as I argue is the case with the relational view, does serve as a good indication of its inadequacy.

I have argued that (R2) does not provide a sufficient criterion to distinguish successful remembering from one form of unsuccessful remembering, i.e., confabulations, as is suggested by Debus's relational view. Before I move on to consider misremembering, let me come back to the relationship between (R1) and confabulations. As I said initially, I think that relationalists can satisfactorily rule out some cases of confabulation by appealing to (R1), as subjects have not experienced the relevant events. The argument I gave so far only provides reasons to deny that (R2) is neither necessary nor sufficient to draw the relevant distinction between successful remembering and confabulation. The problem with placing the burden of the distinction on (R1) is that it undermines the whole motivation for providing a relational account of memory. In Debus's (2008) version of the view, (R2) plays the explanatory role of distinguishing successful remembering from unsuccessful remembering. This is suggested, among other things, by the radical move that Debus makes in favor of disjunctivism. But if, as I argued, (R2) is not required to draw the distinction, the question that poses itself is why give it a central place in one's account of memory, so as to call it a relational view of memory, when, in reality, it is only playing a marginal role in one's analysis of remembering.

But even if we set this worry aside, relationalism would still provide an incomplete account of confabulations. I have assumed throughout my discussion that confabulations are falsidical occurrences of remembering, that is, that they are cases where subjects get things wrong. However, some authors have recently pointed to the possibility of veridical confabulations (Robins 2016a, 2017; Michaelian 2016b; Bernecker 2010), that is, cases where subjects get things right by mere accident. The most intuitive analogy here, as Michaelian (2016b) points out, is to veridi- 
cal perceptual hallucinations (see Lewis 1980). Veridical hallucinations refer to cases where subjects perceive the environment accurately, but the cause of the their perceptual experiences is not the environment, but something else, such as evil scientists or some misfiring happening at the neuronal level. Similarly, veridical confabulations are cases where subjects remember the past correctly, but this is not due to their memories being adequately related to the past, but due to something else, such as the malfunctioning of the underlying mechanisms responsible for memory, or to the occurrence of processes not ordinarily associated with remembering, such as guessing. Assuming that veridical confabulations are possible, and hence that a complete analysis of remembering must be able to distinguish them from falsidical confabulations, simply appealing to (R1) would not provide such a distinction. Both veridical and falsidical confabulations fail to satisfy (R1); and, for this reason, the relational view would mistakenly place these two different occurrences of confabulations under the same category.

To summarize the discussion up to this point, I do not intend the argument above to offer a definitive case against pursuing relational views in relation to confabulations. However, if correct, then it shows that appealing solely to the notion of an experiential relation is not the right way to go.

Before I move forward, I should address one objection that is likely to arise in the context of the introduction of partial experiential relations. Some might argue that, even if there is such a thing as a partial experiential relation, it cannot be used to characterize confabulations appropriately. In particular, the claim is that partial experiential relations cannot explain the phenomenology of confabulation. Like successful remembering, confabulations seemingly make us aware of unique events; however, if they can be partially related to multiple events, as I have suggested, it would follow that it is impossible for us to experience confabulations as involving awareness of unique events. Instead, it would be more plausible to say that they make us aware of a conjunction of two or more events. This would conflict, however, with how we experience confabulations.

This worry can be resolved by noticing that, in partial experiential relations, we become aware of parts or properties of events (Sant'Anna 2018a). For example, the event of my tenth birthday party has different properties, such as "having chocolate cake", "having a meal with my family", and so on. Being fully or wholly experientially related to this event would entail that I would be aware of all the properties instantiated by this event. In contrast, in cases where I am only partially experientially related to this event, I am aware of one or more (but not all) of its properties, e.g., "I remember having chocolate cake". Being aware of this particular property does not, however, make me automatically aware of the event as a whole. So, when confabulations put a subject in multiple partial experiential relations to different events, it is not required that the subject experiences the confabulations as being composed by multiple events. 
Instead, the subject is aware of properties of multiple events that are somehow brought together to consciousness. In a representationalist framework, the equivalent claim here would be that different bits of representational content are brought together to form a new representation of a unique event.

In addition, rather than being incapable of explaining the phenomenology of confabulation, one might argue that, if there is no such thing as a partial experiential relation, relationalists cannot offer a positive account of the phenomenology of confabulation. Because, in confabulations, we are not fully or wholly aware of any events, it would not be possible for us to experience those mental states as making us aware of events at all. One might, of course, retreat to a negative form of disjunctivism here and simply claim that it is not the relationalist's business to explain confabulations, as those are different in kind from successful remembering. ${ }^{17}$ However, motivating a view along these lines is no easy task, so unless relationalists are prepared to offer a positive account of confabulation that does not involve partial experiential relations, the prospects of relationalism will be even dimmer if it does not adopt the notion of partial experiential relations.

\subsection{The problem of misremembering}

I have argued that the relational analysis of remembering faces trouble to distinguish successful remembering from the first form of unsuccessful remembering, which I have called confabulations. I will now consider the second form of unsuccessful remembering, misremembering, which poses another pressing problem for the relational view. The argument proposed in this section is somewhat independent from the argument proposed in 4.1. I will argue that, even if we put the problems pertaining to confabulations aside, the relational view still fails to distinguish between successful remembering and misremembering.

As I discussed before, in cases of misremembering, one is aware of an event that one experienced, but one gets some details of the event wrong. Consider the birthday party example above. Suppose that, instead of remembering having chocolate cake, I remember having strawberry cake. I get all the other details about the event right: I remember my parents and friends being there, I remember it being a rainy day, and etc., but I remember the flavor of my birthday cake wrong. In this case, I am arguably aware of an event that happened, but remember one detail wrong - i.e., the flavor of my birthday cake.

How does the relational view distinguish successful remembering from misremembering? To approach this question, we can employ the same strategy used in the case of confabulations, that is, we can ask whether there are cases of misremembering that satisfy (R1) and (R2). Since the events that are objects of misremembering have been experienced in the past, it seems un-

\footnotetext{
${ }^{17}$ For a similar "negative" strategy to deal with perceptual hallucinations, see Martin (2004); Fish (2009); Brewer (2011)
} 
controversial that (R1) is satisfied. However, and this is where the problem that misremembering poses for the relational view becomes more evident, because those events happened and were experienced, it looks like that (R2) is also satisfied, for subjects stand in the right kind of experiential relation to the events. But, if that is the case, then occurrences of misremembering satisfy the criteria to be classified as successful remembering, which is implausible from the perspective of the relational view.

There is one obvious way for relationalists to avoid this problem. This consists in denying that, when I remember having strawberry cake at my tenth birthday party, I am misremembering a past event that actually happened. Instead, the suggestion is that, precisely because I remember myself having strawberry cake, and not chocolate cake, I am not remembering my tenth birthday party, but some other event that did not happen. Thus, because the event in question did not happen, and hence was not experienced by me, the putative memory does not qualify as an occurrence of successful remembering because the relevant experiential relation fails to obtain. In other words, my putative memory fails to satisfy both (R1) and (R2).

This solution faces three important problems. The first problem is that it will sound counterintuitive to some. As I discussed before, it has been suggested by different researchers that it is not uncommon for our memories to have elements that are not derived from a unique past experience (see, e.g., Michaelian 2011; De Brigard 2014; Cheng and Werning 2016). Thus, it is not surprising that those memories will often be composed by elements that were not present in the original experience. However, it seems too strong to conclude from this that we are systematically failing to remember the events in question. This would mean that, whenever our memories get some detail wrong, which, as De Brigard (2014) points out, happens quite often, the brain mechanisms responsible for memory would be malfunctioning. Since this is an undesirable result, in order to avoid this and other related worries, it seems more reasonable to say that, in misremembering, we are related to the events that happened, but for some reason or another, we get one or more of the details wrong. This would allow us to say that, overall, the system is functioning as it should, while still allowing for error to be present in its outputs, i.e., in the memories produced.

The second problem this solution faces relates to the issues raised in 4.1. We could, in response, point out that, while it might be true that occurrences of misremembering fail to instantiate unique experiential relation to events, nothing would prevent them from instantiating multiple partial experiential relations to different events. Thus, relationalists would need to deal with the same problems raised in the context of confabulations. In other words, they would need to explain how it is possible for unsuccessful remembering to instantiate experiential relations without making the relational view trivial. And finally, the third problem is that, even if we set these worries aside, and grant relationalists that, when I misremember, my putative memory 
relates to an event that is different from my tenth birthday party, the relational view would still provide an incomplete analysis of remembering. The reason is that it would conflate confabulations and misremembering, thus failing to make sense of the differences between these two forms of unsuccessful remembering. In other words, while the relational view might be able to distinguish between successful from unsuccessful remembering, it will not be able to distinguish between forms of unsuccessful remembering because both misremembering and confabulations are understood in terms of the absence of the relevant experiential relation. Therefore, the relational view provides an inappropriate taxonomy of memory errors, and, consequently, an incomplete analysis of remembering.

In response to the third problem, relationalists might want to deny that there is a real distinction between forms of unsuccessful remembering. The claim here would be that there is no real difference between getting some and all of the details of a given event wrong; in other words, the same process is taking place in both cases, i.e., the subject is ultimately unsuccessfully remembering events. Alternatively put, what I have been called misremembering so far is nothing but a less extreme case of confabulation. Thus, if this is right, relationalism is not obliged to provide a taxonomy of memory errors along the lines proposed here.

This move, I think, faces similar problems to the move discussed in 4.1 that attempts to deny that, by definition, there cannot be occurrences of successful remembering that instantiate partial experiential relations. In other words, it runs the risk of forcing our theoretical intuitions into our analysis of the phenomenon in question. While it is not logically incoherent to say that misremembering is nothing but a form of confabulation, this does not seem to reflect how misremembering and confabulations actually happen in the world. As De Brigard (2014) points out, occurrences of what I have called misremembering should not be seen as "bad" products of our memory systems; instead, they are the expected outcomes given how those systems work. Confabulations, in contrast, occur in situations where something has clearly gone wrong, such as when subjects suffer head injuries that lead to some sort of brain damage (see Hirstein 2005; Bernecker 2017; Michaelian 2018 for more discussion). As Michaelian (2016b) points out, while both misremembering and confabulations share the feature of being both erroneous-in his terminology, they are both "inaccurate"-unlike misremembering, which is produced by mechanisms that are working in a reliable manner, confabulations are produced by mechanisms that are working unreliably (see also Hirstein 2005 for a similar account of confabulations). So, it looks like that the workings of the systems responsible for misremembering and confabulation differ in important senses.

Another problem, which is epistemological in nature, is that misremembering, but not confabulation, seems to put us in a position to know at least some things about the past in a reliable manner. In her recent account of misremembering, Robins (2016a) notes that, "[w]hereas 
misrememberings result from the distortion of retained information, confabulations are wholly inaccurate, reflecting no influence of retained information from a particular past event" (2016a, 434). In other words, despite involving distortion, misremembering can still provide us with some useful information about the relevant events because there is some retention of information from the past, which is not the case with confabulations. Thus, as Robins puts it, "[t]he distinction between misremembering and confabulation is particularly important" (2016a, 434).

In summary, the main point is that, if relationalists want to pursue this path, they will need to provide independent reasons, which are not derived from their initial theoretical motivations, to consider misremembering as being just another form of confabulation. It is not clear, however, whether there are such reasons. In fact, recent work on the subject seems to suggest that it is the other way around. In the absence of such reasons, then, relationalism becomes vulnerable to criticism relating to its failure to provide a picture of remembering that is sensitive to research on unsuccessful remembering. So, denying that there is a real distinction between misremembering and confabulation is likely to bring more problems than solutions for relationalists.

\section{Conclusion}

To conclude, I will discuss one worry that might arise in relation to my discussion of unsuccessful remembering and two potential objections to the overall project of the paper. Concerning unsuccessful remembering, one might (rightly) point out that my characterization of unsuccessful remembering implicitly assumes that the erroneous or distorted elements of memory have to be derived from different experiential sources. However, that is not necessarily the case, as the erroneous elements in some forms of misremembering, such as DRM cases, need not derive from any experiential source. ${ }^{18}$ In response, I would like to clarify that, despite focusing on those cases here, I am not committed to the idea that memory errors are necessarily cases where the erroneous elements are derived from experiential sources. Indeed, in DRM cases, it looks like that appealing to non-experiential elements, such as semantic information influencing retrieval, is more adequate to explain the occurrence of those errors. So, my argument here is not meant to apply to those cases. However, when we look at them more closely, it looks like that the relational view will also have trouble to explain the occurrence of those forms of misremembering. The reason why accounting for error in terms of the influence played by semantic information present at the time of retrieval is appealing is that a form of representationalism is assumed beforehand. In other words, because memory is taken to be a representational state, there is no mystery in how the content of semantic memories, or semantic information more generally, can distort the content of episodic memories. This alternative is not, however, available

\footnotetext{
${ }^{18} \mathrm{I}$ 'm grateful to Sarah Robins for calling my attention to this issue.
} 
to relationalists, for it is not clear whether talk of representation can be coherently integrated into their accounts. So, relationalists will also be required to provide an account of those cases in addition to the ones that were discussed here.

Now, the first potential objection that can be raised to the overall argument of the paper is that the relational view is an attempt to characterize successful remembering only, so it is beyond its scope to provide an account of unsuccessful remembering. In response, I would like to point out that, even if we grant that the unique goal of relationalism is to provide an analysis of successful remembering alone, this analysis needs to be capable of distinguishing between successful remembering from other mental states if it is going to be successful. So, even if relationalists cannot provide an account of what distinguishes different forms of unsuccessful remembering, such as misremembering and confabulating, from one another, it needs to explain how successful remembering differs from unsuccessful remembering more generally. However, if I am correct, current relationalist accounts have not been successful in doing that, for as I have shown in Sections 4.1 and 4.2, cases of misremembering and confabulating are wrongly characterized as occurrences of successful remembering by them.

Finally, the second potential objection that can be raised to the overall argument of the paper is that the relational analysis provided here is incomplete and hence that I did not succeed in dismissing relationalism altogether. While I agree that a knockdown argument against relationalism will require a more detailed argument than the one I provided here, I do not think that acknowledging this fact poses a problem for my argument, for it was not the goal of the paper to provide a definite argument against relationalism. Instead, the goal, which is stated from the outset, was to show that unsuccessful remembering raises crucial problems for the relational view of memory. To make the case for this claim, I provided a relational analysis of remembering that is intended to capture the relational account offered by Debus (2008) and showed that, viewed from the perspective of such an analysis, the relational account does not have the required resources to account for memory errors. Thus, my argument does not rule out the possibility of there being alternative relational accounts of remembering that can account for errors, but the burden of developing such accounts is with the relationalist. So, the paper accomplishes its main goal, which is to show that there are important tensions between the most prominent relational account in the literature and research on memory errors.

To summarize, the goal of this paper was to argue that the most prominent version of the relational view of memory in the recent literature, the one developed by Debus (2008), is unable to handle unsuccessful remembering. This has been accomplished by, first, producing a relational analysis of remembering compatible with the relational view defended by Debus (2008), and second, by considering how such analysis of remembering deals with unsuccessful remembering or memory errors. I argued that the relational view, in its current form, cannot properly 
distinguish between successful remembering and different forms of unsuccessful remembering. I did that by distinguishing between two ways in which unsuccessful remembering can happen: misremembering and confabulation. I argued that the requirement for the presence of an experiential relation, as proposed by Debus (2008), is neither necessary nor sufficient for remembering. This is because there are, on the one hand, cases of remembering that do not instantiate the relevant experiential relations, and, on the other hand, cases of confabulation and misremembering that do instantiate the relevant experiential relations. For this reason, if the relational view is to be successful in the context of empirical research on memory; more specifically, in the context of empirical research on memory errors, it will need to provide extra considerations to show how it can deal with such occurrences.

Acknowledgments: I am grateful to Kirk Michaelian, Sarah Robins, Steve James, Cathy Legg, Bill Fish, and Andrew Moore for comments on previous drafts of this paper. I am also grateful to two anonymous referees for their helpful suggestions. This work is supported by the

French National Research Agency in the framework of the "Investissements d'avenir" program (ANR-15-IDEX-02).

\section{References}

Addis, D. R. (2018). Are episodic memories special? On the sameness of remembered and imagined event simulation. Journal of the Royal Society of New Zealand, 48(2-3):64-88. 15

Bernecker, S. (2008). The Metaphysics of Memory. Springer. 3, 5, 6, 8

Bernecker, S. (2010). Memory: A Philosophical Study. Oxford University Press. 7, 17

Bernecker, S. (2015). Memory in analytic philosophy. In Nikulin, D., editor, Memory: A History, pages 298-315. Oxford University Press. 7

Bernecker, S. (2017). A causal theory of mnemonic confabulation. Frontiers in Psychology, 8:1207. 12,21

Brewer, B. (2011). Perception and Its Objects. Oxford University Press. 2, 9, 19

Byrne, A. and Logue, H. (2008). Disjunctivism: Contemporary Readings. MIT Press. 11

Campbell, J. (2002). Reference and Consciousness. Oxford University Press. 2, 5

Cheng, S. and Werning, M. (2016). What is episodic memory if it is a natural kind? Synthese, 193(5):1345-1385. 7, 15, 20 
Cheng, S., Werning, M., and Suddendorf, T. (2016). Dissociating memory traces and scenario construction in mental time travel. Neuroscience \& Biobehavioral Reviews, 60:82-89. 15

Crane, T. (2006). Is there a perceptual relation? In Gendler, T. and Hawthorne, J., editors, Perceptual Experience, pages 126-146. Oxford University Press. 5

Crane, T. and French, C. (2017). The problem of perception. In Zalta, E. N., editor, The Stanford Encyclopedia of Philosophy. Metaphysics Research Lab, Stanford University, Spring 2017 edition. 10

De Brigard, F. (2014). Is memory for remembering? Recollection as a form of episodic hypothetical thinking. Synthese, 191(2):155-185. 15, 20, 21

Debus, D. (2008). Experiencing the past: A relational account of recollective memory. Dialectica, 62(4):405-432. 2, 3, 4, 5, 8, 9, 10, 11, 12, 13, 16, 17, 23, 24

Debus, D. (2010). Accounting for epistemic relevance: A new problem for the causal theory of memory. American philosophical quarterly, 47(1):17-29. 6

Fernández, J. (201x). Memory: A Self-Referential Account. Oxford Universirty Press. Forthcoming. 5

Fish, W. (2009). Perception, Hallucination, and Illusion. Oxford University Press. 2, 9, 11, 19

Genone, J. (2016). Recent work on naive realism. American Philosophical Quarterly, 53(1):125. 5

Hanna, R. (2015). Cognition, Content, and the A Priori: A Study in the Philosophy of Mind and Knowledge. Oxford University Press. 6

Harman, G. (1990). The intrinsic quality of experience. Philosophical Perspectives, 4:31-52. 4

Hirstein, W. (2005). Brain Fiction: Self-Deception and the Riddle of Confabulation. MIT Press. $10,12,21$

Hutto, D. D. and Myin, E. (2017). Evolving enactivism: Basic minds meet content. MIT Press. 5

Hutto, D. D. and Peeters, A. (2018). The roots of remembering: Radically enactive recollecting. In Michaelian, K., Debus, D., and Perrin, D., editors, New Directions in the Philosophy of Memory, pages 97-118. Routledge. 5 
Lewis, D. (1980). Veridical hallucination and prosthetic vision. Australasian Journal of Philosophy, 58(3):239-249. 18

Locatelli, R. and Wilson, K. A. (2017). Perception without representation. Topoi, 36(2):197— 212. 5

Logue, H. (2014). Experiential content and naïve realism: A reconciliation. In Brogaard, B., editor, Does Perception Have Content?, pages 220-241. Oxford University Press. 6

Martin, C. B. and Deutscher, M. (1966). Remembering. Philosophical Review, 75(2):161-96. $7,8,9,14$

Martin, M. (2004). The limits of self-awareness. Philosophical Studies, 120(1):37-89. 2, 5, 8, $9,11,19$

Michaelian, K. (2011). Generative memory. Philosophical Psychology, 24(3):323-342. 14, 15, 20

Michaelian, K. (2016a). Against discontinuism: Mental time travel and our knowledge of past and future events. In Michaelian, K., Klein, S. B., and Szpunar, K. K., editors, Seeing the Future: Theoretical Perspectives on Future-Oriented Mental Time Travel, pages 62-92. Oxford University Press. 3

Michaelian, K. (2016b). Confabulating, misremembering, relearning: The simulation theory of memory and unsuccessful remembering. Frontiers in Psychology, 7:1857. 12, 17, 21

Michaelian, K. (2016c). Mental Time Travel: Episodic Memory and Our Knowledge of the Personal Past. MIT Press. 5, 15

Michaelian, K. (2018). Confabulating as unreliable imagining: In defence of the simulationist account of unsuccessful remembering. Topoi, pages 1-16. 10, 12, 21

Michaelian, K. and Robins, S. K. (2018). Beyond the causal theory? Fifty years after Martin and Deutscher. In Michaelian, K., Debus, D., and Perrin, D., editors, New Directions in the Philosophy of Memory, pages 13-32. Routledge. 7, 9

Michaelian, K. and Sant'Anna, A. (2019). Memory without content? radical enactivism and (post) causal theories of memory. Synthese, pages 1-29. 5

Robins, S. (2018). Mnemonic confabulation. Topoi, pages 1-12. 10, 12

Robins, S. K. (2016a). Misremembering. Philosophical Psychology, 29(3):432-447. 9, 10, 12, $17,21,22$ 
Robins, S. K. (2016b). Representing the past: Memory traces and the causal theory of memory. Philosophical Studies, 173(11):2993-3013. 5, 7, 14

Robins, S. K. (2017). Confabulation and constructive memory. Synthese. Online ahead of print. 12,17

Russell, J. and Hanna, R. (2012). A minimalist approach to the development of episodic memory. Mind \& Language, 27(1):29-54. 6

Sant'Anna, A. (2018a). The hybrid contents of memory. Synthese. Online first. 3, 5, 6, 18

Sant'Anna, A. (2018b). Perception pragmatized: A pragmatic reconciliation of representationalism and relationalism. Philosophia, 46(2):411-432. 6

Sant'Anna, A. and Michaelian, K. (2019). Thinking about events: A pragmatist account of the objects of episodic hypothetical thought. Review of Philosophy and Psychology, 10:187-217. 3,6

Schacter, D. L., Addis, D. R., and Buckner, R. L. (2007). Remembering the past to imagine the future: the prospective brain. Nature Reviews Neuroscience, 8(9):657-661. 15

Schacter, D. L., Addis, D. R., Hassabis, D., Martin, V. C., Spreng, R. N., and Szpunar, K. K. (2012). The future of memory: remembering, imagining, and the brain. Neuron, 76(4):677694. 15

Schellenberg, S. (2010). The particularity and phenomenology of perceptual experience. Philosophical Studies, 149(1):19-48. 6

Schellenberg, S. (2011). Perceptual content defended. Noûs, 45(4):714-750. 6

Siegel, S. (2010). The Contents of Visual Experiences. Oxford University Press. 6

Smith, A. D. (2002). The problem of perception. Harvard University Press. 10

Snowdon, P. (1980). Perception, vision and causation. Proceedings of the Aristotelian Society, 81:175-192. 11

Suddendorf, T. and Corballis, M. C. (1997). Mental time travel and the evolution of the human mind. Genetic, Social, and General Psychology Monographs, 123(2):133-167. 15

Tulving, E. (1972). Episodic and semantic memory. In E.Tulving and Donaldson, W., editors, Organization of Memory, pages 381-402. Academic Press. 3 
Tulving, E. (1985). Elements of Episodic Memory. Oxford University Press. 3

Tulving, E. (2002). Episodic memory: from mind to brain. Annual Review of Psychology, 53(1):1-25. 3

Tye, M. (2000). Consciousness, Color, and Content. MIT Pres. 5 